\title{
Les années italiennes du jeune Einstein
}

\section{La thèse oubliée sur les forces moléculaires}

Christian Bracco $^{(1)}$ (Christian.Bracco@obspm.fr) et Jean-Pierre Provost ${ }^{(2)}$ (Provost@unice.fr)

(1) Syrte (Observatoire de Paris, PSL Research University, CNRS, Sorbonne Univ., UPMC, LNE)

61 avenue de l'Observatoire, 75014 Paris

(2) Professeur retraité, Université de Nice Sophia-Antipolis

Une meilleure compréhension

de la genèse des idées d'Einstein

de 1905 passe par une

reconstruction de l'environnement

scientifique et des modes et lieux

de travail du jeune Albert en Italie,

à Pavie et à Milan, entre 1895

à l'âge de seize ans et 1901 .

Nous portons notre attention

dans cet article sur l'année 1901

et la thèse sur les forces

moléculaires qu'il abandonne,

avant de se tourner vers

la thermodynamique statistique

en 1902.

\section{Michele Besso}

et Mileva Marić, deux témoins de la genèse des idées d'Einstein

Il est bien connu que l'année 1905 voit Albert Einstein, tout juste âgé de vingt-six ans, expert technique au bureau des brevets et de la propriété intellectuelle à Berne, publier plusieurs articles qui feront date dans l'histoire de la physique, comme ceux sur les quanta lumineux et la relativité restreinte. C'est aussi l'année où il soumet sa thèse sur une nouvelle détermination des dimensions moléculaires qu'il applique aussitôt à l'étude du mouvement brownien. 1905 apparaît donc comme une "année miraculeuse " [1], d'autant plus que ses travaux, portant de 1902 à 1904 sur la thermodynamique statistique, ne laissaient pas entrevoir directement cet aboutissement. Or, si ses réflexions cristallisent en 1905, elles ont été l'objet de questionnements antérieurs comme Einstein l'a dit lui-même au sujet de la relativité et des quanta. Quant à sa thèse de 1905, elle prend le contrepied d'une thèse entamée fin 1900 puis abandonnée début 1902, qui portait sur les forces moléculaires en faisant l'hypothèse que la taille des molécules ne jouait pas de rôle.

Pour mieux comprendre ces questionnements, il convient de revenir sur les années de formation du jeune Einstein, qui peuvent être retracées à travers sa correspondance avec Michele Besso, son ami, et Mileva Marić, sa future femme. Rappelons qu'il rejoint fin 1894 sa famille qui se partage entre Pavie et Milan ${ }^{(a)}$ et qu'il intègre en 1896 l'École polytechnique fédérale de Zurich (ETH) dont il sort diplômé en 1900. Nous renvoyons à un

$>>$

\section{Le jeune Albert Einstein en quelques dates}

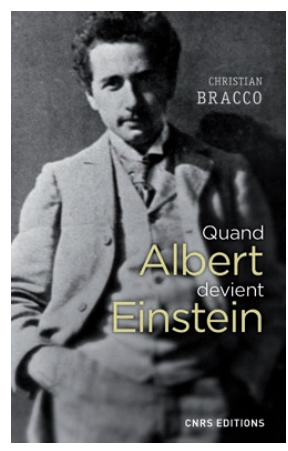

1895 Pavie, tentative d'intégration à l'âge de seize ans de l'ETH de Zurich.

1896 Admission après une année à Aarau pour terminer ses études secondaires.

1900 Diplôme de l'ETH obtenu avec H.-F. Weber sur les phénomènes thermiques.

1900-1901 Travail de thèse sur les forces moléculaires et recherche d'un poste d'assistant.

1896-1901 Séjours réguliers à Milan.

1902 Obtention d'un poste d'expert technique au bureau des brevets de Berne.

1902-1904 Travaux sur les fondements cinétiques et statistiques de la thermodynamique. 
Michele Besso (1873-1955)

Albert le rencontre au moment où il envisage d'entrer à l'ETH de Zurich en 1895 et où Michele en sort. Les deux amis se fréquentent à Aarau et à Zurich entre 1896 et 1899, puis à Milan jusqu'à 1901. Après le décès de son père fin 1901, Michele s'établit à Trieste mais continue d'échanger avec Albert. II le rejoint au bureau des brevets de Berne début 1904.

Michele Besso et son épouse Anna Winteler avant 1898 .

\section{Mileva Marić (1875-1948)}

Jeune fille d'origine serbe qu'Albert épouse en 1903,
Mileva est sa condisciple à l'ETH depuis 1897. Elle est
I'une des rares filles à entreprendre alors des études
scientifiques. Ils se séparent en 1915, après l'installation
d'Albert à Berlin à l'invitation de Planck.
Mileva Marić en 1896.

\section{\〉>}

ouvrage récent [2] pour des précisions sur l'environnement électrotechnique de sa famille en Italie, sur son environnement scientifique et son lien avec la famille Besso, et sur les nombreux sujets de physique qui le préoccupent avant $1902^{(\mathrm{b})}$. Nous nous limitons dans cet article à son intérêt pour la capillarité et à sa première thèse sur les forces moléculaires, abandonnée en février 1902 au moment où il se tourne vers la thermodynamique statistique.

Rappelons que Michele Besso s'est tenu au côté d'Einstein pendant les années qui vont marquer sa consécration et que son rôle concernant la relativité est salué dans les remerciements de l'article de juin $1905^{(\mathrm{c})}$. Si Einstein dira de lui qu'il a été pour ses idées la "meilleure caisse de résonance de toute l'Europe ", Michele se définira, à l'époque de la construction de la théorie de la relativité générale, comme un "pygmée » aux côtés d'un "géant ", mais un "pygmée voyant» [3]. Il apparait dans la correspondance Einstein-Besso [4] que, jusqu'à la fin de sa vie, Besso invitera Einstein, sans succès, à écrire l'histoire de ses idées. Ainsi dès 1923, s'apercevant qu'il a égaré "la collection complète " des travaux de son ami qui "redonnait vie à tant de nos discussions ", il lui propose de mettre cette "collection rare [...] à la portée de tous, en $y$ ajoutant l'essentiel d'un aperçu historique, de la genèse des problèmes [...] " en indiquant "dans l'introduction ce qui, au début de tes recherches, était déjà connu des travaux d'autrui : Boltzmann, Lorentz, ensuite Planck ...". Il conclut : "Je ne cacherai pas au lecteur les fausses routes suivies, car elles sont particulièrement instructives. " La thèse abandonnée de 1902, que nous discutons ci-dessous, en est un exemple.

Les lettres d'Albert à Mileva Marić donnent de précieux aperçus sur son contexte de travail, ses questionnements scientifiques et leur évolution, et permettent à plus d'un siècle d'intervalle de tenter de répondre aux demandes réitérées de Michele Besso. Publiées dans les Collected papers, puis dans un livre [5], elles sont pour la plupart écrites entre 1899 et 1901 à Milan, où sa famille réside, et correspondent aux périodes de vacances semestrielles de l'ETH. On voit Einstein s'interroger par exemple sur la conduction électrique et thermique des métaux ou la nature de la lumière et de l'électricité, ou encore discuter dans une même soirée d'avril 1901 avec Michele à Milan "de la séparation essentielle de l'éther lumineux et de la matière, de la définition du repos absolu, des forces moléculaires, des phénomènes de surface, de la dissociation". Son travail se nourrit d'incessantes lectures, qui suscitent enthousiasme, doutes, idées nouvelles souvent vite remises en question. L'étude des lettres, couplée à une recherche de terrain [2], a permis de préciser ces lectures et le lieu où Albert travaillait.

\section{L'intérêt pour les phénomènes de capillarité, 1895-1900}

En octobre 1900, Einstein souhaite entreprendre une thèse, bien qu'il n'ait pas obtenu de poste d'assistant. Son premier article, rédigé en décembre 1900 et publié en mars 1901 dans les Annalen der Physik [6], porte sur les forces moléculaires déduites des phénomènes de capillarité (voir l'encadré p. 33). On sait que son intérêt pour la capillarité vient en partie des cours qu'il a reçus d'Hermann Minkowski à l'ETH en 1897-1898 et de sa lecture depuis septembre 1899 des travaux de Boltzmann (lettres à Mileva), mais il est aussi en rapport avec la famille Marangoni à Pavie. Le jeune Albert, âgé de seize ans, fait en effet la connaissance à Pavie en 1895 d'Ernestina Marangoni, une jeune femme de dix-neuf ans issue de la bourgeoisie locale, dont l'oncle Carlo Marangoni est spécialiste de ce domaine. Ce dernier formait les enseignants de physique à l'Institut Technique Supérieur de Florence et rendait visite une fois l'an à son frère à la période des vendanges qu'Albert passait avec les Marangoni dans leur villa de Casteggio au sud de Pavie. C'est ainsi, entre 1895 et 1900, que le jeune Einstein a pu se familiariser avec la capillarité en bénéficiant par exemple des explications de Carlo sur les « larmes de vin»! Celui-ci a en effet laissé son nom en physique à l'effet Gibbs-Marangoni qui caractérise un transport de matière entre deux liquides de tensions superficielles différentes; il est cité à trois reprises dans l'article encyclopédique que Minkowski consacre en 1907 à la capillarité(d). 


\section{Milan 1901 et le revirement de la thèse}

\section{La bibliothèque de l'Institut Lombard et Giuseppe Jung}

Si l'on veut comprendre la riche activité scientifique qu'Albert Einstein développe au printemps 1901 à Milan, il faut reconstituer son environnement de travail. Deux lettres à Mileva, datées des 4 et 10 avril, nous apprennent qu'il travaille dans une bibliothèque proche de chez lui et y consulte notamment "une étude de Paul Drude sur la théorie des électrons ». Or il n'y a à cette époque que celle de l'Institut Lombard, académie des sciences et des lettres, qui possède les Annalen der physik où ces articles sont publiés [3]. Créé sur le modèle de l'Institut de France par Napoléon Bonaparte dans le cadre de la République cisalpine, l'Institut Lombard a eu pour premier président Alessandro Volta en 1804. Il joue un rôle central dans le développement de l'industrie et de la recherche italienne à la fin du $\mathrm{XIX}^{\mathrm{e}}$ siècle. L'Institut est situé dans un haut lieu de la culture milanaise, le palais Brera, où se trouvent aussi l'observatoire astronomique, la pinacothèque et la bibliothèque Braidense, le tout à cinq cents mètres de la résidence des Einstein du 21 via Bigli.

Notons que l'accès à la bibliothèque de l'Institut ne pouvait se faire qu'avec l'appui d'un académicien. Or, dans sa recherche d'un poste d'assistant au printemps 1901, Albert mentionne à Mileva le nom de Giuseppe Jung, auquel il a fait remettre son article sur la capillarité par Michele Besso et qui, précise-t-il, " a promis d'écrire pour moi aux professeurs italiens (physiciens) les plus réputés, Righi et Batelli ». Jung, oncle maternel par alliance de Michele, est mathématicien, professeur de statique graphique à l'École polytechnique de Milan depuis 1874 et membre effectif de l'Institut. Albert le présente comme l'une des deux personnes lui assurant une «protection" scientifique à Milan et "l'un des professeurs les plus influents d'Italie ". Comme le montrent les registres d'archives de l'École polytechnique de Milan, il lui a en outre envoyé les tirés-à-part de ses douze premiers articles, jusqu'à 1906 inclus. En 1922, c'est sur proposition de Jung qu'Einstein deviendra membre étranger de l'Institut Lombard, quelques mois avant son prix Nobel [2].

\section{Le Jubilé pour Lorentz et l'article de Max Reinganum}

La présence d'Albert Einstein au palais Brera nous est rapportée par son gendre Rudolf Kayser qui, dans sa biographie, pensait qu'à Milan il "étudiait les arts plastiques et graphiques : [...] les collections de Brera - un monde de beauté classique!". Mais le jeune Einstein devait plutôt y fréquenter la bibliothèque de l'Institut et l'on doit s'intéresser aux sources qui ont pu influencer ses travaux. Le Jubilé pour Lorentz reçu le 31 janvier 1901, qui célèbre le vingt-cinquième anniversaire de la thèse de ce dernier et qui contient une soixantaine de contributions écrites de grands physiciens, nous est apparu comme une source à privilégier. On trouve notamment des indications dans les lettres à Mileva qu'Albert y a certainement lu l'article de Max Planck sur les résonateurs. En avril 1901, la préoccupation principale d'Albert est sa thèse. Si Mileva pensait en décembre 1900 qu'il la soutiendrait à Pâques 1901, celle-ci connaît un brusque revirement. Le 14 avril, Albert fait part à son ami Marcel Grossmann de l'extension de son sujet aux forces moléculaires dans les gaz: "En ce qui concerne la science, j’ai eu quelques idées splendides, qui ne nécessitent maintenant qu'une incubation appropriée. Je suis maintenant convaincu que ma théorie des forces d'attraction atomiques peut aussi être étendue aux gaz, et qu'il sera possible d'obtenir les constantes caractéristiques de presque tous les éléments, sans beaucoup d'efforts. Cela va également amener le problème de la parenté interne entre forces moléculaires et action à distance newtonienne beaucoup plus proche de sa solution. Il est possible que les expériences déjà faites par d'autres avec d'autres objectifs vont suffire pour tester la théorie. Dans ce cas, je vais utiliser tous les résultats existants dans ma thèse de doctorat. "Il écrit en des termes analogues le lendemain à Mileva et ajoute qu'on pourrait aussi avec les "solutions infiniment diluées $[. .$.$] déterminer un grand nombre de c_{\alpha} "$. Une lettre de Mileva en décembre 1901 à son amie Helena Savić confirme que cette thèse a bien été achevée : "Albert a écrit une magnifique étude, qu'il a soumise comme thèse. Il obtiendra probablement son doctorat dans quelques mois [...]. Le sujet porte sur la recherche des forces moléculaires dans les gaz en utilisant différents phénomènes connus."

Le Jubilé pour Lorentz contient l'article de Max Reinganum sur les forces moléculaires dans les gaz faiblement comprimés [7], dont le titre même retient naturellement l'attention [8]. 
Reinganum détermine l'équation d'état des gaz à partir de forces d'attraction "planétaires ", semblables à la gravitation mais en différant par la dépendance avec la distance. D'après lui, la taille des molécules ne joue aucun rôle, de même que dans les processus de dissociation. Ce point de vue marginal est en contradiction avec celui que Boltzmann défend dans ses Leçons sur la théorie des gaz qu'Albert lit avec grand intérêt. Pourtant il l'adopte quand il rappelle le 30 avril 1901 à Mileva : "Je suis très curieux de savoir si nos forces moléculaires conservatives s'appliqueront aux gaz. À condition toutefois que je ne sois pas obligé de faire intervenir la taille des molécules - ce concept mathématiquement si peu clair - dans la formation des trajectoires des molécules qui s'approchent les unes des autres, et que donc chaque molécule puisse être considérée comme un centre de force. " Albert connaît Reinganum (cité par Boltzmann) et écrit même à son patron à Leyde, Heike Kammerlingh Onnes, pour candidater à un poste d'assistant (deux jours avant l'envoi de sa lettre à Grossmann).

Rappelons aussi qu'en avril 1901, Albert discute avec Michele de la dissociation. Il a certainement étudié le chapitre du tome II des Leçons qui présente une théorie cinétique de la dissociation moléculaire (par exemple $2 \mathrm{HI} \rightarrow \mathrm{H}_{2}+\mathrm{I}_{2}$ ), capable de reproduire les résultats obtenus sur une base purement entropique par Gibbs et Planck cités explicitement. Pour Boltzmann, la liaison chimique est décrite de façon continue par une énergie d'interaction proportionnelle aux volumes de recouvrement de «domaines relativement petits situés sur la surface des atomes ». Boltzmann insiste sur le caractère directionnel des interactions et rappelle en conclusion que c'est en vain qu'il " $a$ recherché autrefois à rendre compte [...] de la façon dont les molécules se comportent ", si on les considère " comme des points matériels " avec "des forces d'attraction ne faisant pas intervenir les forces de percussion". Einstein a aussi pu voir dans ce rappel un défi théorique le conduisant à s'intéresser à Reinganum, dont il dira en 1911, reconnaissant implicitement la "fausse route» suivie, que les articles "sont plutôt négligés".

\section{2 : I'abandon de la thèse et la continuation d'une lecture critique de Boltzmann}

Fin décembre 1901, Albert s'apprête à soutenir sa thèse, dont il a remis le manuscrit à son directeur, le professeur Alfred Kleiner de l'université de Zurich. Il ne doute alors pas de son succès et écrit à Mileva que par rapport à ses collègues de promotion il aura fini son travail le premier. Mais un mois plus tard, il demande le remboursement de ses droits d'inscription à l'université, suite certainement au rejet du manuscrit (aujourd'hui disparu). Ce rejet pourrait être lié à l'hypothèse faite sur l'absence de rôle de la taille des molécules, et la thèse de 1905 sur leurs dimensions, toujours avec Kleiner, en serait en quelque sorte une confirmation a posteriori.

Une publication [9] dans le domaine des solutions diluées, qu'Albert avait mentionnée à Mileva en avril 1901, est très probablement liée au travail remis à Kleiner. Il s'agit en effet d'un article d'électrochimie où les coefficients $c_{\alpha}$ sont introduits pour les ions et les solvants; mais ne pouvant en déduire leurs valeurs en raison de multiples hypothèses, Einstein termine par des excuses pour n'avoir présenté "qu'un maigre projet pour des recherches laborieuses sans contribuer en quoi que ce soit à leur solution expérimentale." Le tournant d'Einstein vers la thermodynamique statistique est probablement, comme la "fausse route » de 1901, lié à une lecture critique mais incomplète de Boltzmann. En effet, par les lettres à Mileva on apprend qu'en novembre 1901 il lit Planck et son approche entropique

(a) L'entreprise électrotechnique Einstein, Garrone \& Cie est établie à Pavie de 1894 à 1896, où la famille réside 11 via Ugo Foscolo avant de s'installer définitivement à Milan.

(b) L'étude de ces environnements a permis en particulier de mieux comprendre sa première tentative d'entrer à l'ETH en 1895 sans avoir ni le diplôme ni l'âge requis, ainsi que la rédaction concomitante d'un article étonnant sur l'état de l'éther dans un champ magnétique.

(c) Moins connu est le rappel que Besso adresse à Einstein dans une lettre de 1928 à propos des quanta : "j'ai été ton public pendant les années 1904 et 1905 ; en t'aidant à rédiger tes communications sur le problème des quanta je t'ai privé d'une partie de ta gloire, mais en revanche, je t'ai procuré un ami, Planck ». L'allusion à 1904 fait probablement référence à la toute fin de l'article d'Einstein [11], où les fluctuations d'énergie sont appliquées au rayonnement du corps noir [8].

(d) Carlo Marangoni a été aussi l'assistant du recteur de l'université de Pavie, le physicien Giovanni Cantoni, connu pour avoir donné dès 1867 une interprétation cinétique du mouvement brownien.

des processus naturels, et qu'en février 1902 il a rendu à Kleiner un travail se rapportant au livre de Boltzmann. L'origine probable de ce tournant est que Boltzmann, en renvoyant au début du tome I des Leçons le lecteur à des travaux antérieurs qu'Albert n'a pas dû lire [10], ne fait qu'esquisser les liens entre calcul des probabilités, irréversibilité, fonction $H$, entropie et loi de répartition des énergies. Le jeune Einstein aura besoin d'écrire trois articles pour clarifier ces liens. À la clé, par application dans le troisième [11] des fluctuations d'énergie au rayonnement du corps noir, il y aura les quanta, $c f$. note $(c)$.

\section{Références}

1. J.J. Stachel, Einstein's Miraculous Year, Five papers that changed the face of physics (2005), Princeton University Press.

2. C. Bracco, Quand Albert devient Einstein (2017), CNRS Editions, Paris.

$3 \cdot$ C. Bracco, "Einstein and Besso: From Zürich to Milan", Rend. Scienze, 148 (2014) 285-322. www.ilasl.org/index.php/Scienze/article/view/178

4. P. Speziali, Albert Einstein, Michele Besso Correspondance 1903-1955 (1979), Hermann, Paris.

5• A. Einstein et M. Marić, Lettres d'amour et de sciences (1993), Éditions du Seuil, Paris.

6. A. Einstein, Annal. der Physik 4 (1901) 513-523, traduit dans "Conclusions drawn from the phenomena of capillarity", The Collected Papers of Albert Einstein, vol. 2. [CPAE 2], pp. 9-21, J. Stachel et al. eds., Princeton University Press (1989). http://einsteinpapers.press.princeton.edu/

7• M. Reinganum, "Molekuläre Anziehung in schwach comprimierten Gasen", Arch. Néerl. Sci. Ex. et Nat., 5 (1900) [Jubilé pour Lorentz], 574-582.

8. C. Bracco et J.P. Provost, "Perspectives on Einstein's scientific work in Milan", Proc. $14^{\text {th }}$ Marcel Grossmann meeting, Bianchi, Jantzen et Ruffini eds., (2017), pp. 3337-3341, World Scientific, Singapore. https://arxiv.org/abs/1508.04917.

9. A. Einstein, Annal. der Phys. 8 (1902) 798-814, traduit dans "On the thermodynamic theory of the difference in potentials between metals and fully dissociated solutions of their salts and on an electrical method for investigating molecular forces" [CPAE 2, pp. 22-40].

10. C. Cercignani, Ludwig Boltzmann: The Man Who Trusted Atoms (1998), Oxford University Press.

11• A. Einstein, Annal. der Physik 14 (1904) 354-362, traduit dans "On the general molecular theory of heat" [CPAE 2, pp. 98-108]. 